\title{
Clinical impact of anatomical resection on long-term outcomes after hepatectomy for primary solitary hepatocellular carcinoma with or without preoperative positron emission tomography positivity
}

\author{
Chan Woo Cho ${ }^{1}$, Jong Man Kim², Beom-Hui Lee ${ }^{1}$, Dong-Shik Lee ${ }^{1}$, Sung-Su Yun ${ }^{1}$, Gyu-Seong Choi ${ }^{2}$, \\ Jae-Won Joh ${ }^{2}$ \\ ${ }^{1}$ Department of Surgery, Yeungnam University College of Medicine, Daegu, Korea; ${ }^{2}$ Department of Surgery, Samsung Medical Center, \\ Sungkyunkwan University School of Medicine, Seoul, Korea \\ Contributions: (I) Conception and design: CW Cho, JM Kim; (II) Administrative support: SS Yun, JW Joh; (III) Provision of study materials or \\ patients: All authors; (IV) Collection and assembly of data: BH Lee, JM Kim; (V) Data analysis and interpretation: CW Cho, DS Lee, GS Choi; (VI) \\ Manuscript writing: All authors; (VII) Final approval of manuscript: All authors. \\ Correspondence to: Professor Jong Man Kim, MD, PhD. Department of Surgery, Samsung Medical Center, Sungkyunkwan University School of \\ Medicine, \#81 Irwon-ro, Gangnam-gu, Seoul 06351, Korea. Email: jongman94.kim@samsung.com.
}

\begin{abstract}
Background: There is little evidence indicating that anatomical resection (AR) is associated with improved survival in patients with solitary hepatocellular carcinoma (HCC) who were preoperatively evaluated by positron emission tomography (PET). The aim of our study was to compare the oncologic outcomes of AR in PET-positive versus PET-negative patients with HCC.

Methods: From January 2007 to September 2015, 259 patients with preoperative PET underwent hepatectomy as the primary treatment for solitary HCC. Patients were divided into four groups according to PET uptake and hepatectomy type [AR or non-anatomical resection (NAR)]: Group 1 (PET-negative and AR, n=62); Group 2 (PET-negative and NAR, n= 46); Group 3 (PET-positive and AR, n=100); Group 4 (PET-positive and NAR, $\mathrm{n}=51$ ).

Results: PET positivity was associated with higher protein induced by vitamin $\mathrm{K}$ antagonist-II $(\mathrm{P}=0.025)$, lager tumor size $(\mathrm{P}=0.05)$, microvascular invasion (MVI) $(\mathrm{P}=0.012)$, and portal vein invasion $(\mathrm{P}=0.031)$. In Kaplan-Meier analysis for RFS, Group 1 showed remarkable difference from Group 3 and Group 4 ( $\mathrm{P}=0.045$, $\mathrm{P}=0.023$, respectively). In the PET-positive subgroup with $\mathrm{HCC}$ under $3 \mathrm{~cm}$, AR was associated with better RFS than NAR $(\mathrm{P}=0.016)$.

Conclusions: A combination of AR and PET negativity showed good prognosis in long-term outcomes. Finally, AR can decrease the risk of tumor recurrence in patients with a solitary PET-positive HCC less than $3 \mathrm{~cm}$.
\end{abstract}

Keywords: Hepatocellular carcinoma (HCC); anatomical resection (AR); positron emission tomography (PET)

Submitted Feb 14, 2020. Accepted for publication Sep 11, 2020.

doi: $10.21037 /$ atm-20-1583

View this article at: http://dx.doi.org/10.21037/atm-20-1583

\section{Introduction}

Liver resection is a curative treatment option for patients with solitary hepatocellular carcinoma (HCC) and preserved liver function $(1,2)$. Anatomical resection (AR) including the area covered by the tumor-feeding portal vein was preferred over non-anatomical resection (NAR) as a surgical technique to prevent potential micrometastasis surrounding the tumor because HCC tends to invade 
intrahepatic vascular structures and is often close to the portal vein $(3,4)$. Despite establishment of improved surgical techniques, tumor recurrence is still a major concern for the long-term survival of patients who undergo liver resection. The recurrence rate of HCC after curative hepatectomy reaches up to $70 \%$ within 5 years $(5,6)$. Tumor size, serum alpha-fetoprotein (AFP), protein induced by vitamin $\mathrm{K}$ antagonist-II (PIVKA-II), c-reactive protein, alkaline phosphatase, neutrophil to lymphocyte ratio (NLR), and neutrophil to monocyte ratio (NMR) were reported as risk factors of tumor recurrence that can be preoperatively evaluated (7-10). Microvascular invasion (MVI) is also considered an independent prognostic factor predicting HCC recurrence or poor survival in HCC after curative resection, but it is difficult to assess prior to operation. Recently, increasing evidence demonstrates that preoperative $18 \mathrm{~F}$-fluorodeoxyglucose $\left({ }^{18} \mathrm{~F}\right.$-FDG) positron emission tomography (PET)/computed tomography (CT) can predict MVI and early recurrence after liver resection (11-14).

In this study, we assessed whether combined AR with preoperative PET negativity was associated with positive prognostic factors. We also investigated whether AR achieves better oncologic outcomes than NAR regarding preemptive control of MVI among patients with primary solitary HCC and positive PET findings. We present the following article in accordance with the STROBE reporting checklist (available at http://dx.doi.org/10.21037/atm-201583).

\section{Methods}

\section{Study design and population}

This study was conducted on patients who underwent liver resection for solitary HCC diagnosed by radiologic imaging at Yeungnam University Medical Center and Samsung Medical Center in Korea between January 2007 and September 2015. HCC patients meeting the following criteria were excluded: history of previous treatments for HCC such as liver resection, transarterial chemoembolization, percutaneous ethanol injection, radiofrequency ablation (RFA), and radiation therapy; concurrent intraoperative RFA; combined HCC and cholangiocarcinoma on pathology; loss to follow-up after liver resection; no PET/CT performed for preoperative assessment; and multiple HCCs on pathology. As a result, this study included 259 treatment-naïve HCC patients who underwent preoperative PET/CT and subsequent curative liver resection as a primary treatment at two institutions. Baseline demographic, laboratory, pathologic, and surgical data were retrospectively collected and analyzed from the electronic medical record. Patients were divided into four groups according to preoperative PET uptake and liver resection type (AR or NAR): Group 1 (PET-negative and AR); Group 2 (PET-negative and NAR); Group 3 (PETpositive and AR); Group 4 (PET-positive and NAR). The study was conducted in accordance with the Declaration of Helsinki (as revised in 2013). The institutional review boards of Samsung Medical Center (number 2016-08-161) and Yeungnam University Medical Center (number 201902-041) approved this study. Individual informed consent was waived because of the retrospective nature of the study.

\section{Study criteria}

The main purpose of our study was to compare recurrencefree survival (RFS) and overall survival (OS) among four groups and to identify the combined impact of AR and preoperative PET positivity on long-term oncologic outcomes in patients with primary solitary HCC. Liverrelated mortality was defined as death from HCC recurrence after hepatectomy, as well as death caused by hepatectomyrelated complications including hepatic failure, sepsis due to bile leakage, and postoperative bleeding. AR was defined as complete resection of an anatomic region demarcated by preceding ischemia, along with division of the Glisson associated with tumor location. With solitary HCC located peripherally or presenting with exophytic growth, NAR was performed when patients required limited resection due to insufficient liver function or remnant liver volume. By comparing pre- and postoperative CT of each patient, the resection type was evaluated to conform to the definition of AR identifying the location of the tumor, the extent of the resected liver parenchyme, and the ligated portal pedicle. Major hepatectomy was defined as removal of 3 or more liver segments (15). The histologic grade of HCC was evaluated according to Edmonson-Steiner grade (E-S grade) as 'well differentiated' (grade I), 'moderately differentiated' (grade II), or 'poorly differentiated' (grade III, IV) (16).

\section{${ }^{18}$ F-FDG PET/CT}

All ${ }^{18}$ F-FDG PET/CT imaging was performed using Discovery VCT scanners and Discovery STe scanners (GE 
Medical Systems, Milwaukee, WI, USA) at Yeungnam University Medical Center and Samsung Medical Center, respectively. PET/CT scans were acquired after a single FDG injection. Patients fasted for 6 hours before the ${ }^{18} \mathrm{~F}$-FDG injection (serum glucose level $<140 \mathrm{mg} / \mathrm{dL}$ ). FDG dose was corrected for body mass index, and approximately $5.5 \mathrm{MBq} / \mathrm{kg}$ of FDG was administered intravenously. Uptake of ${ }^{18} \mathrm{~F}$-FDG on PET/CT was visually interpreted as positive or negative by comparing the foci of increased metabolic activity between normal surrounding tissues and tumor tissue (12).

\section{Statistical analysis}

Continuous data were presented as mean $[ \pm$ standard deviation (SD)]. Categorical data were described in numbers and percentages. Statistical analysis was conducted using an independent-sample $t$-test or a Mann-Whitney test for continuous values and a Chi-square test or Fisher's exact test for categorical values, especially when expected cell frequencies were below five. RFS and liver-related OS rates were analyzed via the Kaplan-Meier method. The log-rank test was used to compare survival curves produced from two groups. Univariate and multivariate analyses for risk factors affecting HCC recurrence or liverrelated mortality following hepatectomy were conducted using a Cox proportional hazard model. $\mathrm{P}$ values below 0.05 were considered statistically significant. Data handling and analysis were performed using the Statistical Package for Social Science for Windows ${ }^{\mathrm{TM}} 22.0$ release (SPSS Inc., Chicago, IL).

\section{Results}

\section{Baseline demographic, laboratory, pathologic, and surgical factors}

Table 1 shows the baseline characteristics of HCC patients with PET-positive and PET-negative findings. The main (92.6\% vs. 97.3\%) etiology in both groups was hepatitis $\mathrm{B}$ virus (HBV). PIVKA-II $(\mathrm{P}=0.025)$, tumor size $(\mathrm{P}=0.05)$, MVI $(\mathrm{P}=0.012)$, and portal vein invasion $(\mathrm{P}=0.031)$ were significantly higher in the PET-positive group than in the PET-negative group. There were no significant differences in demographic factors such as age, sex, ChildTurcotte-Pugh (CTP) class; laboratory factors such as white blood cells, NLR, NMR, platelet count, aspartate aminotransferase (AST), alanine aminotransferase (ALT), total bilirubin, prothrombin time international normalized ratio (PT INR), albumin, AFP, and indocyanine green retention rate at 15 minutes (ICG R15); pathologic factors such as Edmonson-Steiner grade, Glisson capsule invasion, bile duct invasion, intrahepatic metastasis, multicentric occurrence, tumor-free margin, and cirrhosis; or surgical factors such as laparoscopic approach, AR, and major hepatectomy.

\section{Impact of ${ }^{18}$ F-FDG PET/CT positivity on long-term oncologic outcomes}

The mean follow-up duration of the entire cohort was $42.2 \pm 22.2$ months. The 1-, 3-, and 5-year RFS rates were $68.0 \%, 59.6 \%$, and $51.5 \%$ in the PET-positive group, respectively, and $85.0 \%, 68.2 \%$, and $59.8 \%$ in the PETnegative group. The RFS rate in the PET-negative group was higher than in the PET-positive group, although this trend was not statistically significant $(\mathrm{P}=0.055$; Figure $1 A)$. The 1-, 3-, and 5-year liver-related OS rates were 94.0\%, $89.0 \%$, and $84.8 \%$, respectively, in the PET-positive group and $100 \%, 93.1 \%$, and $85.7 \%$ in the PET-negative group. However, these tendencies of liver-related OS were not significantly different between the groups $(\mathrm{P}=0.337$; Figure $1 B)$.

\section{Risk factors for disease-free survival and liver-related mortality}

Univariate and multivariate analyses for risk factors affecting HCC recurrence and liver-related mortality are presented in Tables 2 and 3, respectively. Multivariate analysis revealed that higher E-S grade, MVI, intrahepatic metastasis, and multicentric occurrences were significantly associated with HCC recurrence $(\mathrm{P}=0.001, \mathrm{P}=0.037, \mathrm{P}=0.004$, and $\mathrm{P}=0.001$, respectively; Table 2). Independent risk factors affecting liver-related mortality were determined to be higher E-S grade, Glisson capsule invasion, MVI, and multicentric occurrence $(\mathrm{P}=0.013, \mathrm{P}=0.001, \mathrm{P}=0.002$, and $\mathrm{P}<0.001$, respectively; Table 3). Neither preoperative PET positivity nor AR was an independent factor of HCC recurrence and liver-related mortality.

\section{Impacts of ${ }^{18}$ Preoperative PET uptake and AR on long- term oncologic outcomes}

Four groups divided according to preoperative PET uptake 
Table 1 Demographic, laboratory, pathologic, and surgical factors of preoperative PET-negative and PET-positive patients who underwent liver resection as primary treatment for hepatocellular carcinoma

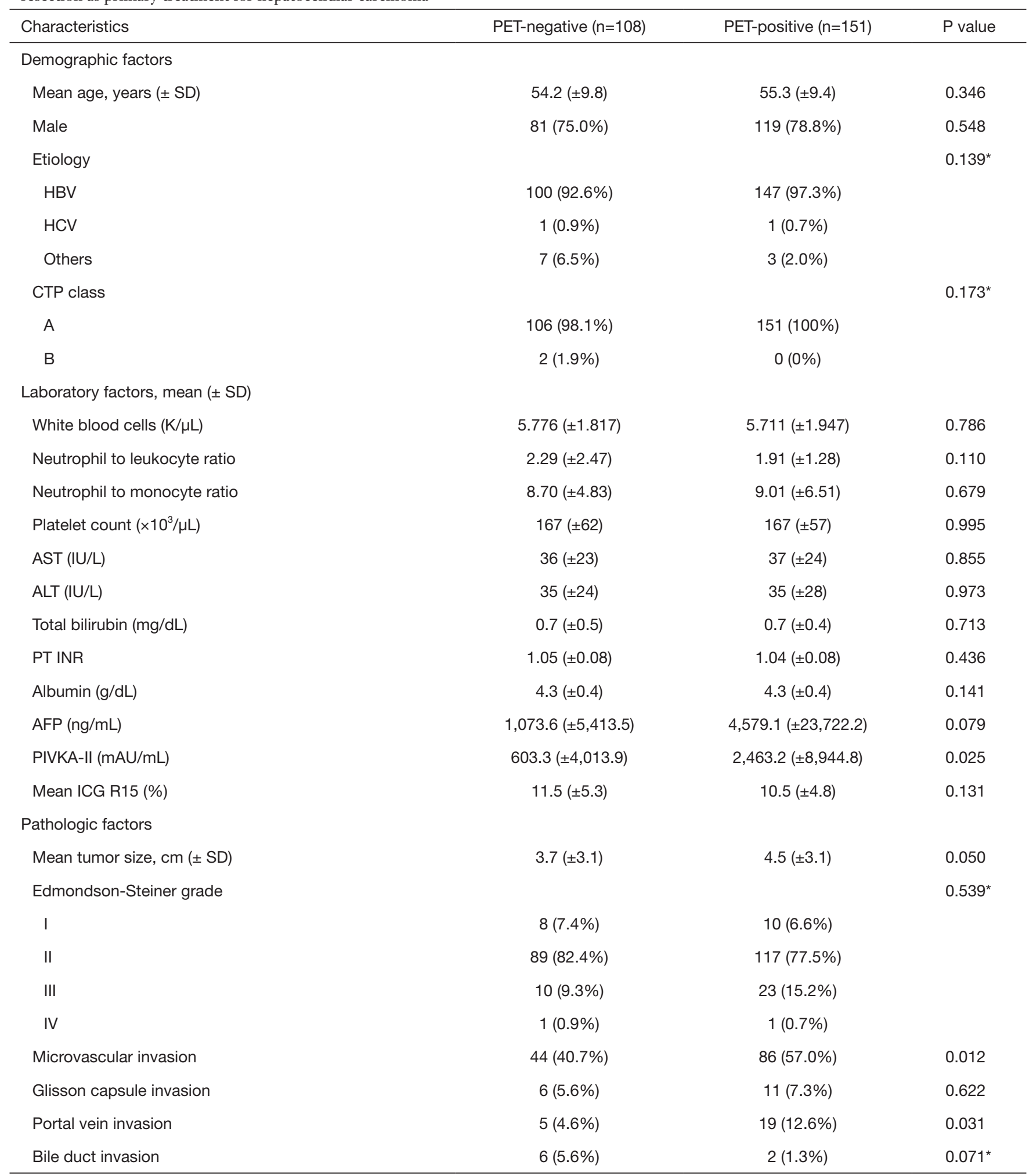

Table 1 (continued) 
Table 1 (continued)

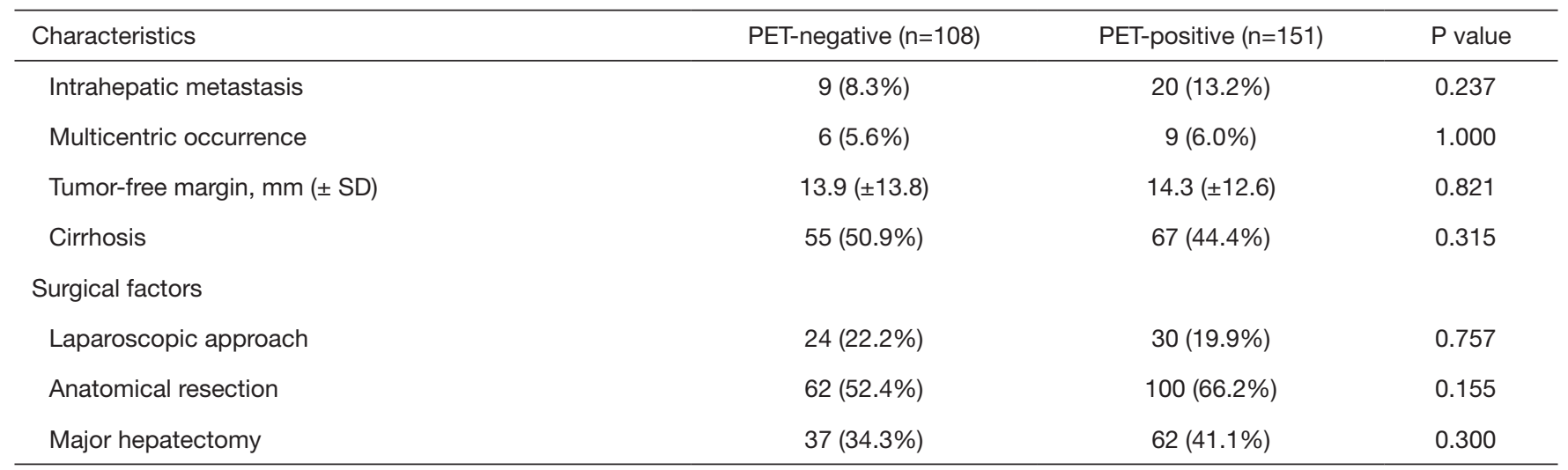

*, Fisher exact test. AFP, alpha-fetoprotein; ALT, alanine aminotransferase; AST, aspartate aminotransferase; CTP, Child-Turcotte-Pugh; HBV, hepatitis B; HCV, hepatitis C; ICG R15, indocyanine green retention rate at 15 minutes; INR, international normalized ratio; PET, positron emission tomography; PIVKA-II, proteins induced by vitamin K antagonist-II; PT, prothrombin time; SD, standard deviation.
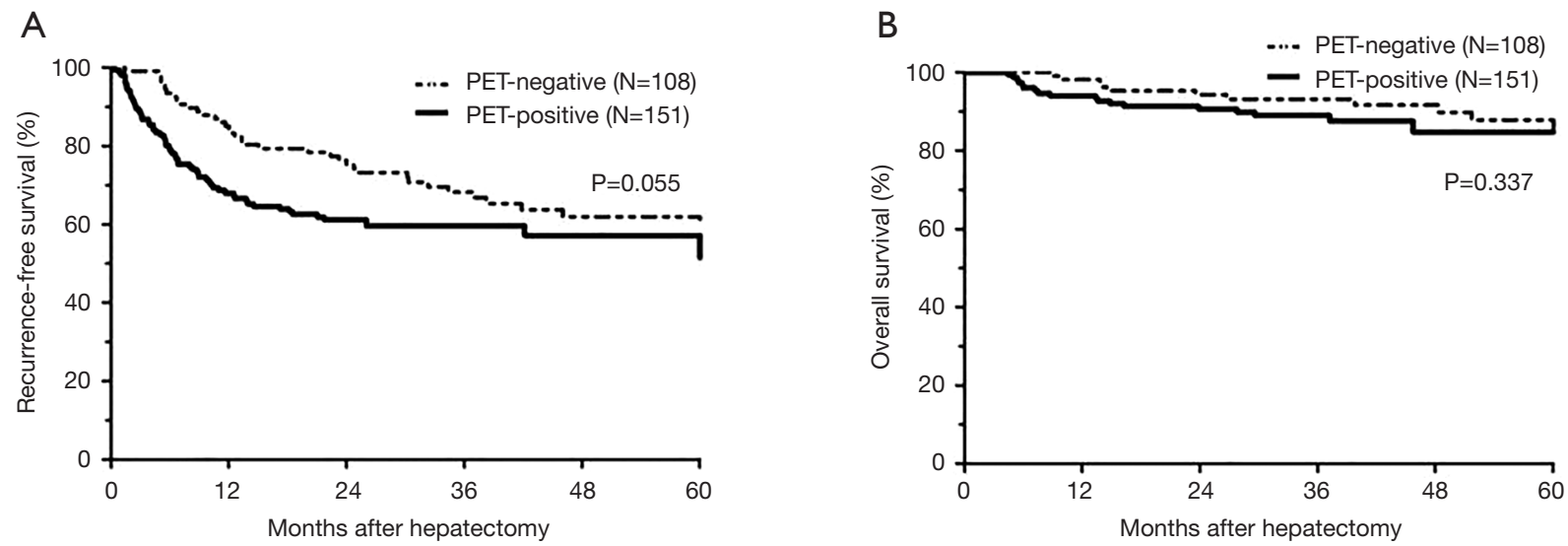

Figure 1 Recurrence-free survival (A) and overall survival (B) of PET-positive and PET-negative groups. PET, positron emission tomography.

and AR did not show statistically significant differences in pathologic factors including E-S grade, Glisson capsule invasion, MVI, intrahepatic metastasis, and multicentric occurrence $(\mathrm{P}=0.387, \mathrm{P}=0.614, \mathrm{P}=0.057, \mathrm{P}=0.480$, and $\mathrm{P}=0.659$, respectively; Table 4). Kaplan-Meier analysis revealed that the RFS and OS of AR were not significantly different from those of NAR among PET-negative groups $(\mathrm{P}=0.100$, Figure $2 A$ and $\mathrm{P}=0.142$, Figure $2 B$, respectively) and among PET-positive groups $(\mathrm{P}=0.743$, Figure $2 C$ and $\mathrm{P}=0.630$, Figure $2 D$, respectively). In Kaplan-Meier analysis among four groups, Group 1 (PET-negative and AR) showed remarkably better RFS than Group 3 (PET-positive and $\mathrm{AR}$ ) and Group 4 (PET-positive and NAR) ( $\mathrm{P}=0.045$, $\mathrm{P}=0.023$, respectively; Figure 3). In subgroup analysis based on tumor size, Group 1 patients with HCC over $3 \mathrm{~cm}$ showed significantly better RFS than Group 2 ( $\mathrm{P}=0.047$, Figure 4), and Group 3 patients with HCC under $3 \mathrm{~cm}$ had remarkably better RFS than Group $4(\mathrm{P}=0.016$, Figure 4). There was no significant difference among subgroups in Kaplan-Meier analysis for OS (Figure 5).

\section{Discussion}

MVI is an independent predictor of HCC recurrence and is related to poor outcome and low survival rates after liver resection $(17,18)$. Therefore, detection of MVI is important to predict the prognosis of patients with HCC at the beginning of treatment. In previous conventional 
Table 2 Univariate and multivariate analyses of risk factors for HCC recurrence

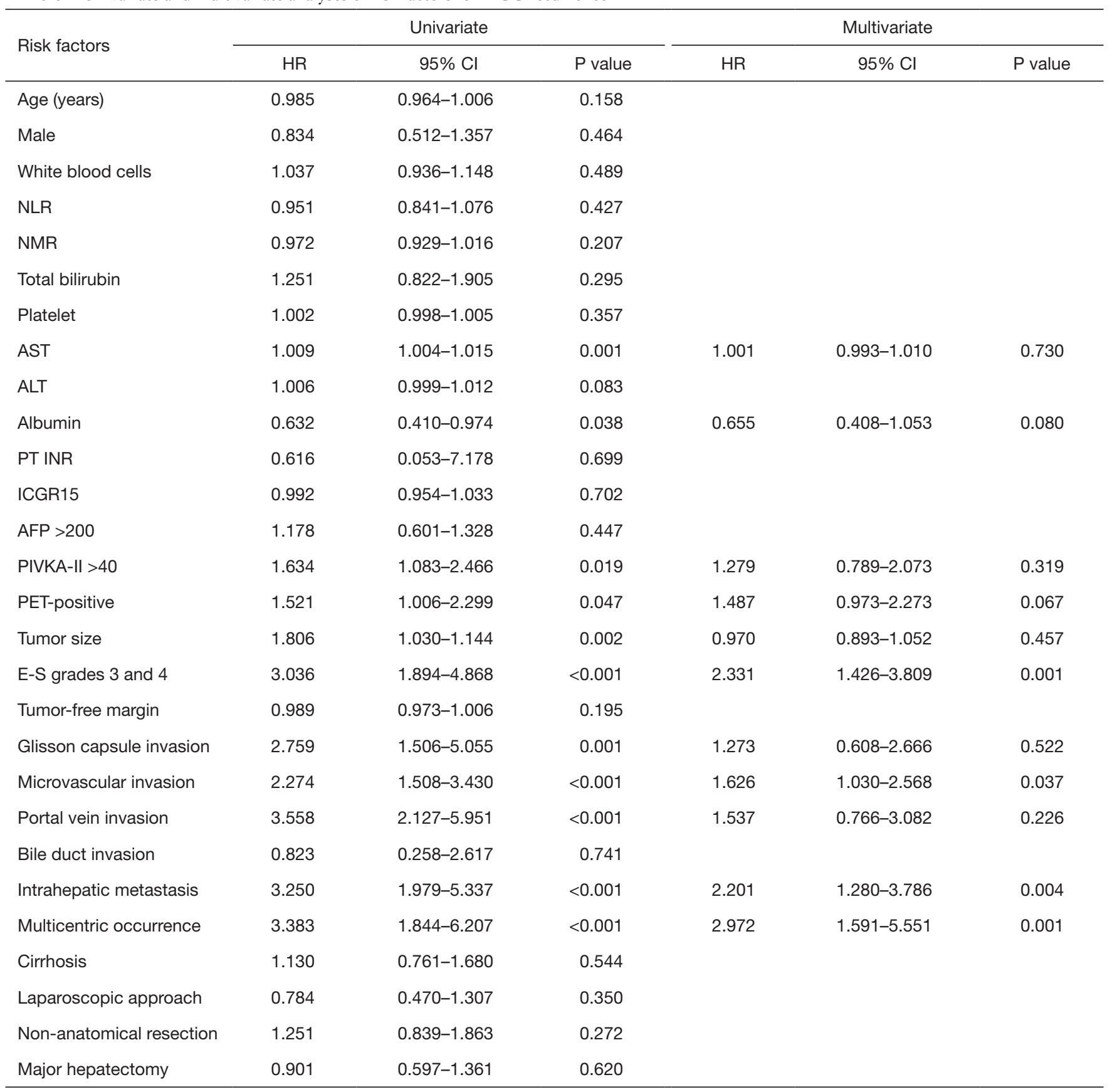

AFP, alpha-fetoprotein; ALT, alanine aminotransferase; AST, aspartate aminotransferase; E-S grade, Edmonson-Steiner grade; HR, hazard ratio; INR, international normalized ratio; ICG, indocyanine green; NLR, neutrophil to leukocyte ratio; NMR, neutrophil to monocyte ratio; PET, positron emission tomography; PIVKA-II, proteins induced by vitamin K antagonist-II; PT, prothrombin time.

imaging studies, MVI is indirectly predicted based on capsule disruption, irregular tumor margin, and peritumoral enhancement (17). In a recent study, Kim et al. reported that the presence of peritumoral hypointensity on hepatobiliary phase of Gadoxetic acid-enhanced magnetic resonance imaging (MRI) showed high specificity (93.2\%) and a positive predictive value of $88.5 \%$. Kim et al. concluded that peritumoral hypointensity on hepatobiliary phase of 
Table 3 Univariate and multivariate analyses of risk factors for liver-related mortality

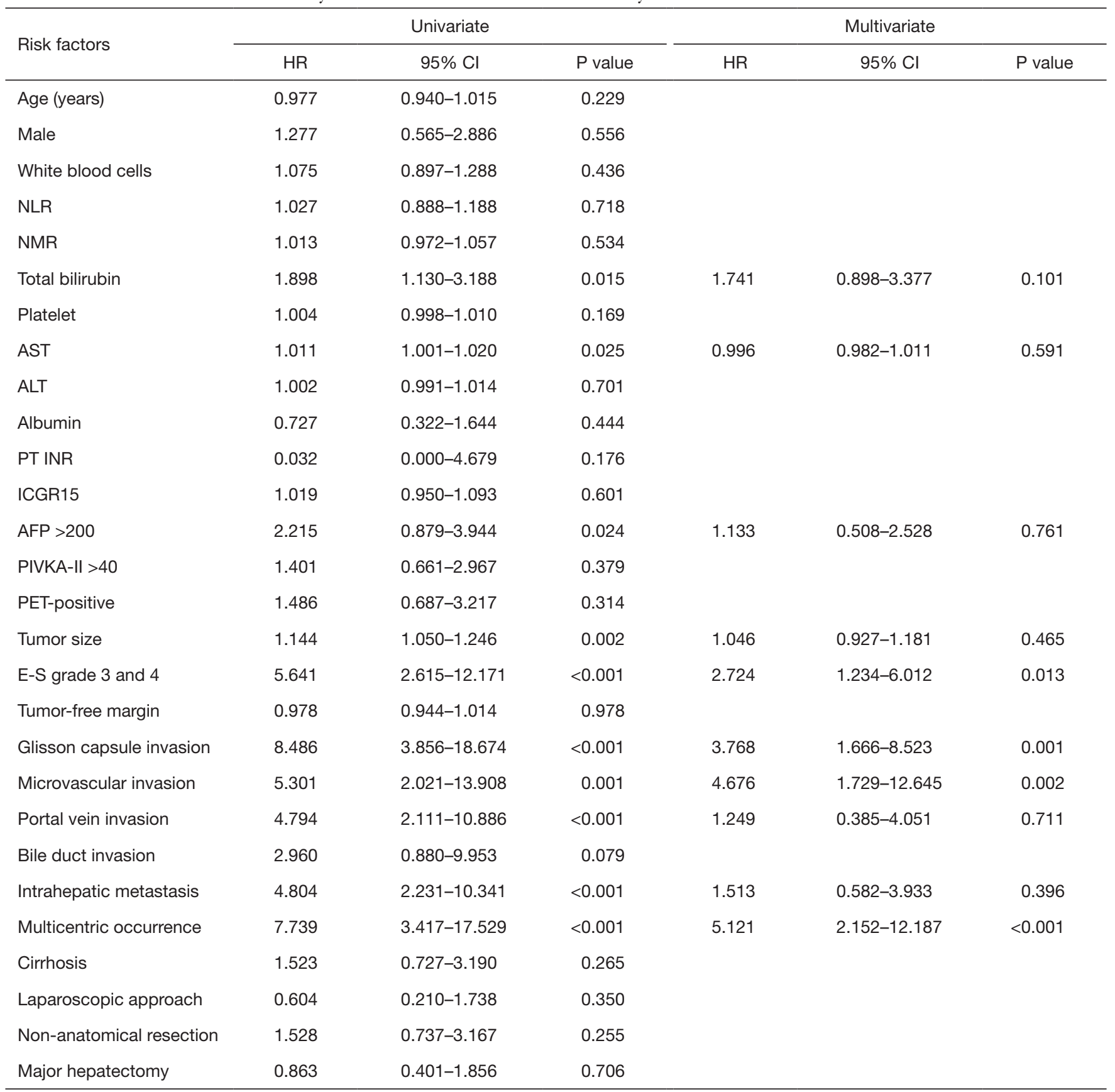

AFP, alpha-fetoprotein; ALT, alanine aminotransferase; AST, aspartate aminotransferase; E-S grade, Edmonson-Steiner grade; HR, hazard ratio; INR, international normalized ratio; ICG, indocyanine green; NLR, neutrophil to leukocyte ratio; NMR, neutrophil to monocyte ratio; PET, positron emission tomography; PIVKA-II, proteins induced by vitamin K antagonist-II; PT, prothrombin time.

gadoxetic acid-enhanced MRI can be useful preoperative predictor of MVI in HCC patients (19). Tumor markers including AFP and PIVKA-II are also adopted to evaluate the biological aggressiveness of HCC prior to surgical resection. PIVKA-II is more sensitive and specific than AFP because it is associated with tumor growth rate, increased cellular proliferation, infiltrative pattern, and vascular invasion. However, these time-dependent laboratory tests 
Table 4 Pathologic factors of groups divided according to preoperative PET uptake and anatomical resection

\begin{tabular}{|c|c|c|c|c|c|}
\hline Characteristics & $\begin{array}{l}\text { Group } 1(\mathrm{n}=62), \\
\text { PET(-)/AR }\end{array}$ & $\begin{array}{c}\text { Group } 2(n=46), \\
\text { PET(-)/NAR }\end{array}$ & $\begin{array}{c}\text { Group } 3(n=100), \\
\operatorname{PET}(+) / A R\end{array}$ & $\begin{array}{c}\text { Group } 4(n=51), \\
\text { PET }(+) / \text { NAR }\end{array}$ & $P$ value \\
\hline Mean tumor size, $\mathrm{cm}( \pm \mathrm{SD})$ & $4.1( \pm 3.4)$ & $3.3( \pm 2.5)$ & $4.6( \pm 3.0)$ & $4.3( \pm 3.2)$ & 0.308 \\
\hline E-S grade 3 and 4 & $7(11.3 \%)$ & $4(8.7 \%)$ & $18(18 \%)$ & $6(11.8 \%)$ & 0.387 \\
\hline Tumor-free margin, mm $( \pm S D)$ & $17.0( \pm 16.4)$ & $9.7( \pm 7.8)$ & $16.9( \pm 13.9)$ & $9.2( \pm 7.5)$ & 0.863 \\
\hline Portal vein invasion & $3(4.8 \%)$ & $2(4.3 \%)$ & $12(12 \%)$ & $7(13.7 \%)$ & $0.190^{*}$ \\
\hline Bile duct invasion & $5(8.1 \%)$ & $1(2.2 \%)$ & $2(2 \%)$ & $0(0 \%)$ & 0.077 \\
\hline Intrahepatic metastasis & $4(6.5 \%)$ & $5(10.9 \%)$ & $12(12 \%)$ & $8(15.7 \%)$ & 0.480 \\
\hline
\end{tabular}

*, Fisher exact test. AR, anatomical resection; E-S grade, Edmonson-Steiner grade; PET, positron emission tomography; NAR, nonanatomical resection; SD, standard deviation.

A

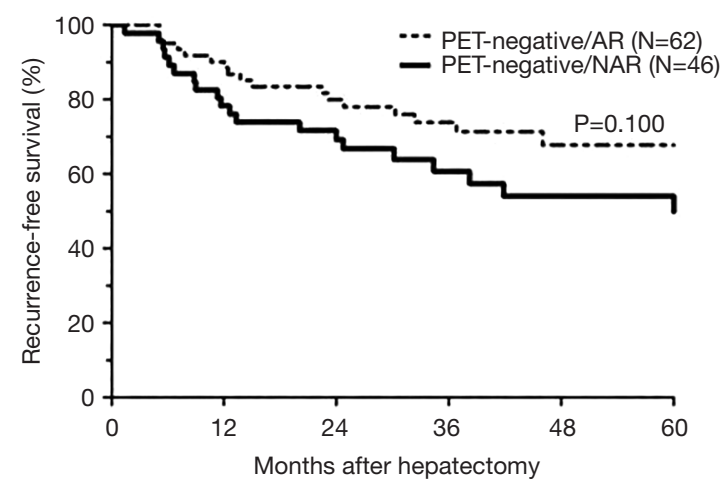

C

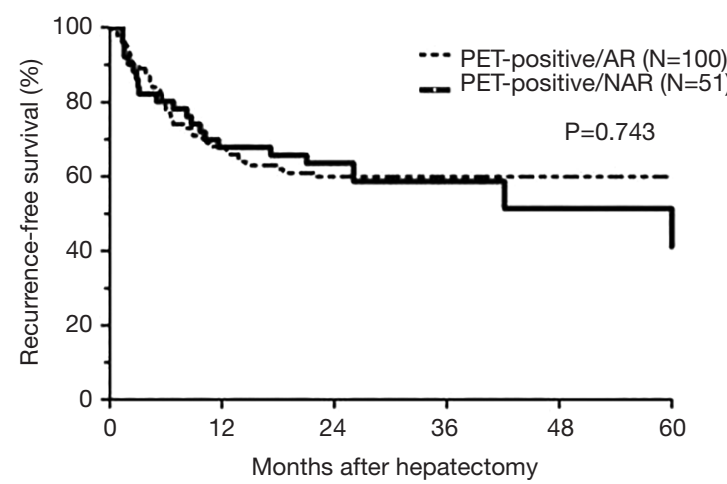

B

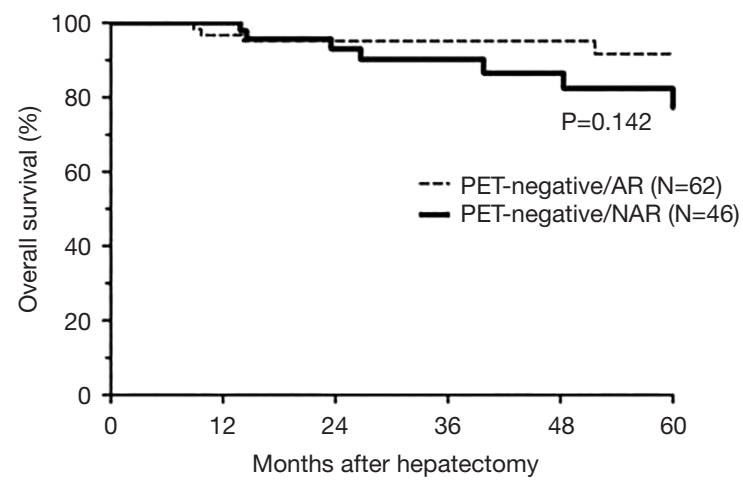

D

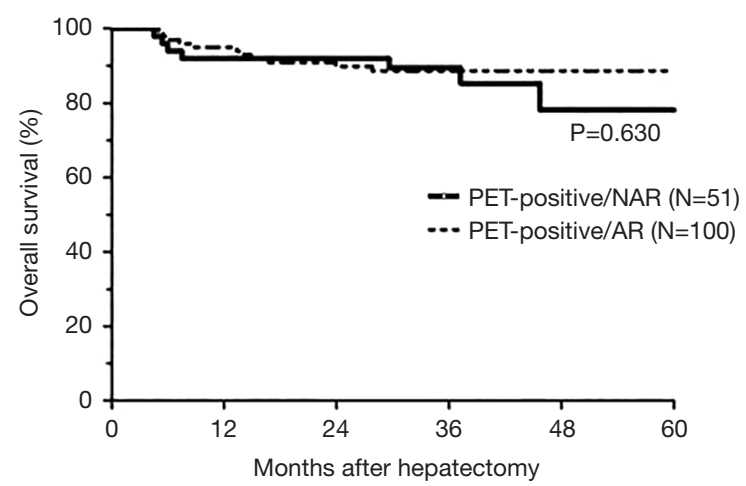

Figure 2 Recurrence-free survival (A) and overall survival (B) of Group 1 and Group 2. Recurrence-free survival (C) and overall survival (D) of Group 3 and Group 4. 
A

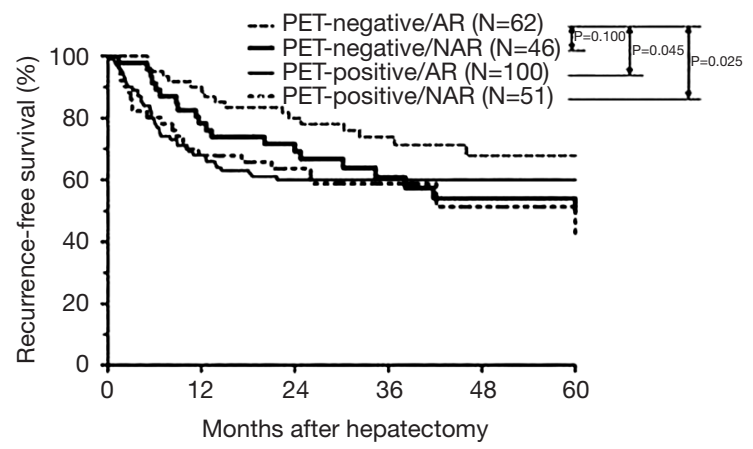

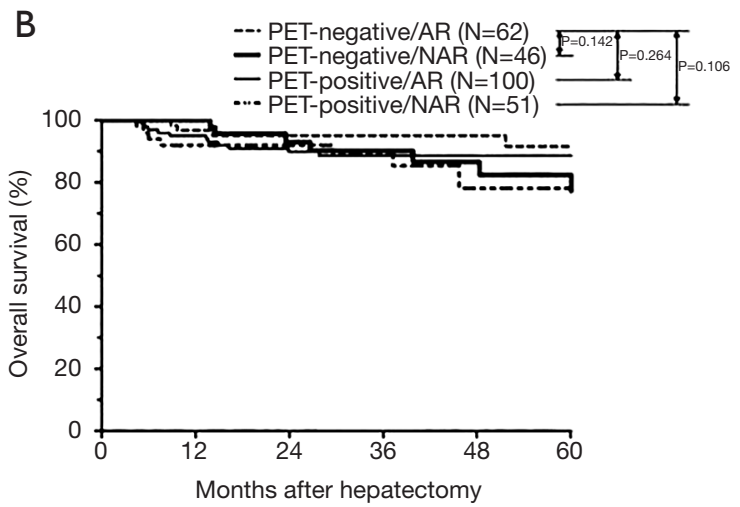

Figure 3 Recurrence-free survival (A) and overall survival (B) of four groups.
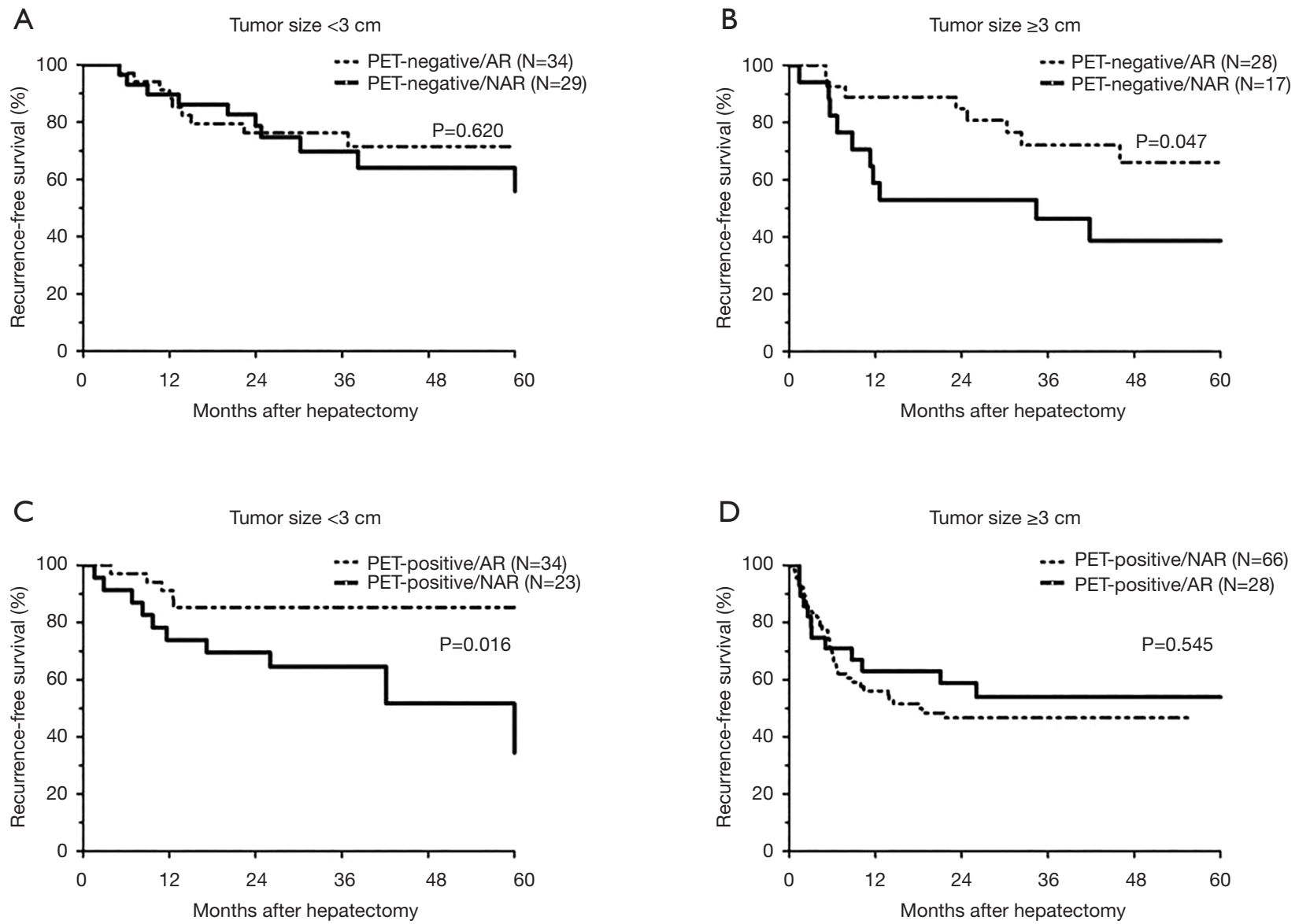

Figure 4 Recurrence-free survival of Group 1 and Group 2 based on tumors less than $3 \mathrm{~cm}$ (A) and more than $3 \mathrm{~cm}$ (B). Recurrence-free survival of Group 3 and Group 4 based on tumors less than $3 \mathrm{~cm}(\mathrm{C})$ and more than $3 \mathrm{~cm}(\mathrm{D})$. 

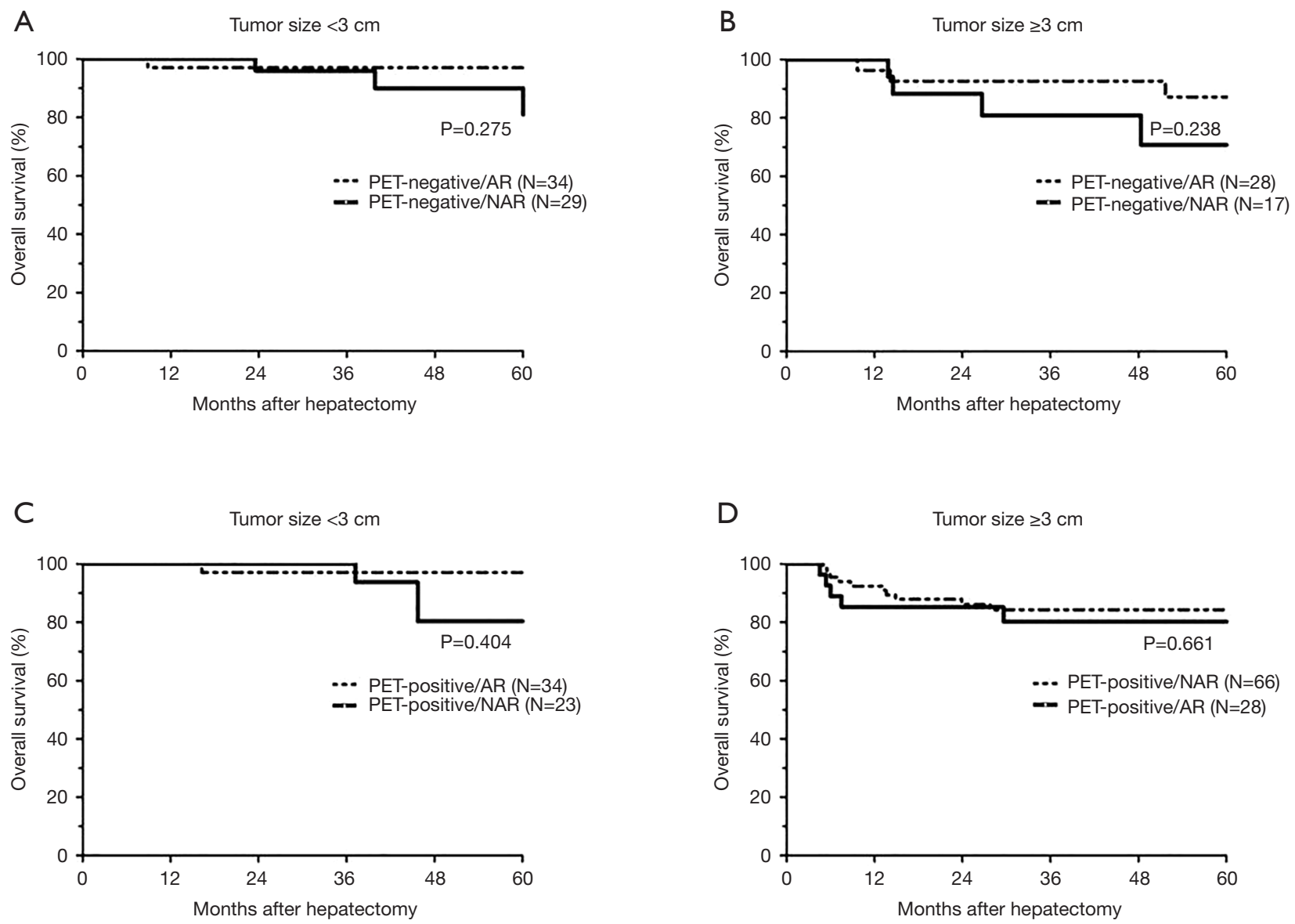

Figure 5 Overall survival of Group 1 and Group 2 based on tumors less than $3 \mathrm{~cm}$ (A) and more than $3 \mathrm{~cm}$ (B). Overall survival of Group 3 and Group 4 based on tumors less than $3 \mathrm{~cm}(\mathrm{C})$ and more than $3 \mathrm{~cm}(\mathrm{D})$.

cannot determine whether or not MVI has occurred, and they only assess the effects of treatment or whether the disease is stationary or progressing.

Since ${ }^{18}$ F-FDG PET/CT shows relatively high physiologic liver uptake and variable uptake in HCC resulting from increased glucose-6-phosphatase activity in normal liver tissues, it has limited use for primary diagnosis of HCC (20). Previous studies have already shown that high standard uptake value (SUV) on PET was significantly associated with high-grade tumor differentiation and was a poor prognostic factor following liver resection for HCC. Lin et al. concluded that the ratio of maximal tumor SUV to mean normal liver SUV of ${ }^{18} \mathrm{~F}$-FDG PET/CT was an independent predictor of MVI (21). Thus, increasing evidence has accumulated demonstrating that positive PET findings can predict MVI $(11,17,22)$ based on the close correlation between MVI and poor tumor grade (23-25).
In the present study, patients with PET-positive HCC tended to have significantly higher PIVKA-II $(\mathrm{P}=0.025)$, portal vein invasion $(\mathrm{P}=0.031)$, and $\mathrm{MVI}(\mathrm{P}=0.012)$ than patients with PET negative HCC (Table 1). This implies that positive PET findings reflect more aggressive HCC biology. However, in multivariate analysis, our study failed to prove that $\mathrm{PET}$ positivity was an independent prognostic factor for HCC recurrence and liver-related mortality. Kaplan-Meier analysis for RFS identified a tendency for higher relapse in the PET-positive group $(\mathrm{P}=0.055$, Figure $1 A$ ).

$\mathrm{AR}$ is preferred for HCC that tends to invade into tumor-feeding vascular structures and theoretically can achieve better oncologic outcomes than NAR. However, there is little clinical evidence that AR is superior to NAR in the long-term survival of HCC patients with preserved liver function $(26,27)$. A recent multicenter-based collaboration 
study using propensity score matching concluded that AR decreases HCC recurrence and improves OS in patients with a primary solitary HCC $<5.0 \mathrm{~cm}$ in diameter (28). In real clinical situations, AR is not applicable to all patients with HCC because of poor liver function, unfavorable tumor location, or insufficient remnant liver volume. Therefore, it is important to make surgical plans according to preoperative assessment for vascular invasion by tumor.

We analyzed long-term oncologic outcomes of AR versus NAR in patients with solitary HCC who were preoperatively evaluated by PET/CT uptake as a surrogate marker of vascular invasion. As shown in Figure 2, AR and NAR in the PET-negative group did not show any significant differences in RFS $(\mathrm{P}=0.100)$ and $\mathrm{OS}(\mathrm{P}=0.142)$. However, the RFS of PET-negative HCC patients who underwent $\mathrm{AR}$ was significantly higher than that of PETpositive HCC patients who underwent $\mathrm{AR}(\mathrm{P}=0.045)$ and NAR $(\mathrm{P}=0.023)$. This result supports that AR for PETnegative HCC might be associated with positive prognosis to prevent recurrence after hepatectomy for solitary HCC. Both MVI and tumor size are known to reflect oncologic properties. MVI is confirmed by invasion on pathologic study, but our study proved that PET positivity can predict the presence of MVI without tissue confirmation. When tumor size and PET positivity were subdivided, AR had better RFS than NAR. For example, AR had better RFS than NAR in the subgroup with HCC over $3 \mathrm{~cm}$ and PET negativity (Figure $4 B$ ) than in the subgroup with HCC under $3 \mathrm{~cm}$ and PET positivity (Figure 4C). In other words, in HCC patients whose tumors are too aggressive or too mild, AR may not be superior to NAR. Therefore, although there is selection bias due to the nature of the retrospective study, it can be said that this study has clinical implications to help the surgeon choose the resection type (AR vs. NAR) by assessing the biological characteristics of the tumor shown in preoperative tests including PET.

A major limitation of our study is the inability to determine PET positivity according to the cut-off value using SUV. Instead of measuring SUV, we visually interpreted the presence of FDG uptake to compare the difference in metabolic activity between tumor and normal liver tissue. In addition, our study had a retrospective design with a relatively short follow-up period. There could be potential selection bias due to inclusion of patients with solitary HCC who underwent ${ }^{18} \mathrm{~F}-\mathrm{FDG}$ PET/CT prior to hepatectomy. Further prospective studies with long-term follow-up in a larger cohort are necessary to confirm our results.
In conclusion, we identified that ${ }^{18} \mathrm{~F}$-FDG uptake of HCC on preoperative PET predicts MVI in hepatectomy patients. In addition, preoperative PET/CT can provide useful clinical information about prognosis after liver resection for HCC, reflecting aggressiveness and tumor differentiation. Although AR and PET negativity were not independent factors affecting HCC recurrence, a combination of AR and PET negativity might indicate good prognosis in long-term outcomes. Finally, AR decreases the risk of tumor recurrence in patients with a solitary PETpositive HCC less than $3 \mathrm{~cm}$.

\section{Acknowledgments}

This work was supported by the 2019 Yeungnam University Research Grant.

Funding: None.

\section{Footnote}

Reporting Checklist: The authors have completed the STROBE reporting checklist. Available at http://dx.doi. org/10.21037/atm-20-1583

Data Sharing Statement: Available at http://dx.doi. org/10.21037/atm-20-1583

Peer Review File: Available at http://dx.doi.org/10.21037/ atm-20-1583

Conflicts of Interest: All authors have completed the ICMJE uniform disclosure form (available at http://dx.doi. org/10.21037/atm-20-1583). The authors have no conflicts of interest to declare.

Ethical Statement: The authors are accountable for all aspects of the work in ensuring that questions related to the accuracy or integrity of any part of the work are appropriately investigated and resolved. The study was conducted in accordance with the Declaration of Helsinki (as revised in 2013). The study protocol was approved by the institutional review boards of Samsung Medical Center (number 2016-08-161) and Yeungnam University Medical Center (number 2019-02-041). Individual informed consent was waived because of the retrospective nature of the study.

Open Access Statement: This is an Open Access article distributed in accordance with the Creative Commons 
Attribution-NonCommercial-NoDerivs 4.0 International License (CC BY-NC-ND 4.0), which permits the noncommercial replication and distribution of the article with the strict proviso that no changes or edits are made and the original work is properly cited (including links to both the formal publication through the relevant DOI and the license). See: https://creativecommons.org/licenses/by-nc-nd/4.0/.

\section{References}

1. Marrero JA, Kulik L, Sirilin C, et al. Diagnosis, staging, and management of hepatocellular carcinoma: 2018 practice guidance by the american association for the study of liver diseases. Hepatology 2018;68:723-50

2. Chedid MF, Kruel C, Pinto M, et al. Hepatocellular carcinoma: diagnosis and operative management. Arq Bras Cir Dig 2017;30:272-8

3. Kaibori M, Ishizaki $M$, Matsui K, et al. Predictors of microvascular invasion before hepatectomy for hepatocellular carcinoma. J Surg Oncol 2010;102:462-8.

4. Makuuchi M, Hasegawa H, Yamazaki S. Ultrasonically guided subsegmentectomy. Surg Gynecol Obstet 1985;161:346-50.

5. Imamura $\mathrm{H}$, Matsuyama $\mathrm{Y}$, Tanaka $\mathrm{E}$, et al. Risk factors contributing to early and late phase intrahepatic recurrence of hepatocellular carcinoma after hepatectomy. J Hepatol 2003;38:200-7.

6. Kwon SK, Yun SS, Kim HJ, et al. The risk factors of early recurrence after hepatectomy in hepatocellular carcinoma. Ann Surg Treat Res 2014;86:283-8.

7. Kim JM, Kwon CH, Joh JW, et al. The effect of alkaline phosphatase and intrahepatic metastases in large hepatocellular carcinoma. World J Surg Oncol 2013;11:40.

8. Kim SH, Moon DB, Kim WJ, et al. Preoperative prognostic values of alpha-fetoprotein (AFP) and protein induced by vitamin $\mathrm{K}$ absence or antagonist-II (PIVKAII) in patients with hepatocellular carcinoma for living donor liver transplantation. Hepatobiliary Surg Nutr 2016;5:461-9.

9. Mano Y, Shirabe K, Yamashita Y, et al. Preoperative neutrophil-to-lymphocyte ratio is a predictor of survival after hepatectomy for hepatocellular carcinoma: a retrospective analysis. Ann Surg 2013;258:301-5.

10. Yang T, Zhu J, Zhao L, et al. Lymphocyte to monocyte ratio and neutrophil to lymphocyte ratio are superior inflammation-based predictors of recurrence in patients with hepatocellular carcinoma after hepatic resection. J Surg Oncol 2017;115:718-28.
11. Lim C, Salloum C, Chalaye J, et al. 18F-FDG PET/CT predicts microvascular invasion and early recurrence after liver resection for hepatocellular carcinoma: A prospective observational study. HPB (Oxford) 2019;21:739-47.

12. Kim JM, Kwon CHD, Joh JW, et al. Prognosis of preoperative positron emission tomography uptake in hepatectomy patients. Ann Surg Treat Res 2018;94:183-9.

13. Ahn SG, Kim SH, Jeon TJ, et al. The role of preoperative $[18 \mathrm{~F}]$ fluorodeoxyglucose positron emission tomography in predicting early recurrence after curative resection of hepatocellular carcinomas. J Gastrointest Surg 2011;15:2044-52.

14. Hatano E, Ikai I, Higashi T, et al. Preoperative positron emission tomography with fluorine-18-fluorodeoxyglucose is predictive of prognosis in patients with hepatocellular carcinoma after resection. World J Surg 2006;30:1736-41.

15. Allard MA, Adam R, Bucur PO, et al. Posthepatectomy portal vein pressure predicts liver failure and mortality after major liver resection on noncirrhotic liver. Ann Surg 2013;258:822-9; discussion 829-30.

16. Edmondson HA, Steiner PE. Primary carcinoma of the liver: a study of 100 cases among 48,900 necropsies. Cancer 1954;7:462-503.

17. Cuccurullo V, Di Stasio GD, Mazzarella G, et al. Microvascular Invasion in HCC: The Molecular Imaging Perspective. Contrast Media Mol Imaging 2018;2018:9487938.

18. Lim KC, Chow PK, Allen JC, et al. Microvascular invasion is a better predictor of tumor recurrence and overall survival following surgical resection for hepatocellular carcinoma compared to the Milan criteria. Ann Surg 2011;254:108-13.

19. Kim AY, Sinn DH, Jeong WK, et al. Hepatobiliary MRI as novel selection criteria in liver transplantation for hepatocellular carcinoma. J Hepatol 2018;68:1144-52.

20. Haug AR. Imaging of primary liver tumors with positronemission tomography. Q J Nucl Med Mol Imaging 2017;61:292-300.

21. Lin CY, Liao CW, Chu LY, et al. Predictive Value of 18F-FDG PET/CT for Vascular Invasion in Patients With Hepatocellular Carcinoma Before Liver Transplantation. Clin Nucl Med 2017;42:e183-e187.

22. Kornberg A, Freesmeyer M, Barthel E, et al. 18F-FDGuptake of hepatocellular carcinoma on PET predicts microvascular tumor invasion in liver transplant patients. Am J Transplant 2009;9:592-600.

23. Esnaola NF, Lauwers GY, Mirza NQ, et al. Predictors of microvascular invasion in patients with hepatocellular 
carcinoma who are candidates for orthotopic liver transplantation. J Gastrointest Surg 2002;6:224-32; discussion 232.

24. Tamura S, Kato T, Berho M, et al. Impact of histological grade of hepatocellular carcinoma on the outcome of liver transplantation. Arch Surg 2001;136:25-30; discussion 31.

25. Okazumi S, Isono K, Enomoto K, et al. Evaluation of liver tumors using fluorine-18-fluorodeoxyglucose PET: characterization of tumor and assessment of effect of treatment. J Nucl Med 1992;33:333-9.

Cite this article as: Cho CW, Kim JM, Lee BH, Lee DS, Yun SS, Choi GS, Joh JW. Clinical impact of anatomical resection on long-term outcomes after hepatectomy for primary solitary hepatocellular carcinoma with or without preoperative positron emission tomography positivity. Ann Transl Med 2020;8(21):1377. doi: 10.21037/atm-20-1583
26. Kaibori M, Matsui Y, Hijikawa T, et al. Comparison of limited and anatomic hepatic resection for hepatocellular carcinoma with hepatitis C. Surgery 2006;139:385-94.

27. Tanaka K, Shimada H, Matsumoto C, et al. Anatomic versus limited nonanatomic resection for solitary hepatocellular carcinoma. Surgery 2008;143:607-15.

28. Kaibori M, Kon M, Kitawaki T, et al. Comparison of anatomic and non-anatomic hepatic resection for hepatocellular carcinoma. J Hepatobiliary Pancreat Sci 2017;24:616-26. 